\title{
Improved Broadband Liner Optimization Applied to the Advanced Noise Control Fan
}

\author{
Douglas M. Nark* and Michael G. Jones ${ }^{\dagger}$ \\ NASA Langley Research Center, Hampton, VA 23681-2199 \\ Daniel L. Sutliff ${ }^{\ddagger}$ \\ NASA Glenn Research Center, Cleveland, $\mathrm{OH} 44212$ \\ Earl Ayle ${ }^{\S}$ and Fumitaka Ichihashi ${ }^{\text {If }}$ \\ Hexcel Corporation, Casa Grande, AZ
}

\begin{abstract}
The broadband component of fan noise has grown in relevance with the utilization of increased bypass ratio and advanced fan designs. Thus, while the attenuation of fan tones remains paramount, the ability to simultaneously reduce broadband fan noise levels has become more desirable. This paper describes improvements to a previously established broadband acoustic liner optimization process using the Advanced Noise Control Fan rig as a demonstrator. Specifically, in-duct attenuation predictions with a statistical source model are used to obtain optimum impedance spectra over the conditions of interest. The predicted optimum impedance information is then used with acoustic liner modeling tools to design liners aimed at producing impedance spectra that most closely match the predicted optimum values. Design selection is based on an acceptance criterion that provides the ability to apply increased weighting to specific frequencies and/or operating conditions. Constant-depth, double-degree of freedom and variable-depth, multi-degree of freedom designs are carried through design, fabrication, and testing to validate the efficacy of the design process. Results illustrate the value of the design process in concurrently evaluating the relative costs/benefits of these liner designs. This study also provides an application for demonstrating the integrated use of duct acoustic propagation/radiation and liner modeling tools in the design and evaluation of novel broadband liner concepts for complex engine configurations.
\end{abstract}

\section{Nomenclature}

c

\section{sound speed}

liner core depth

annular duct height (outer radius - inner radius)

free space wave number

axial liner length

circumferential mode number

total number of frequency/flow condition combinations in optimization

weighting factor utilized in impedance design cost function

\footnotetext{
${ }^{*}$ Research Scientist, Structural Acoustics Branch, Research Directorate, AIAA Associate Fellow

${ }^{\dagger}$ Senior Research Scientist, Structural Acoustics Branch, Research Directorate, AIAA Associate Fellow

¥Aerospace Engineer, Acoustics Branch, AIAA Associate Fellow

${ }^{\S}$ Senior Technical Fellow, Research and Technology Division

IIResearch Engineer Acoustic \& Thermal, Research and Technology Division
} 
Symbols:

$\begin{array}{ll}\zeta & \text { normalized acoustic impedance }(\zeta=\theta+i \chi) \\ \theta & \text { acoustic resistance normalized by } \rho c \\ \mu & \text { mean value } \\ \rho & \text { fluid density } \\ \sigma & \text { standard deviation } \\ \tau & \text { hub-to-tip ratio (inner radius/outer radius) } \\ \chi & \text { acoustic reactance normalized by } \rho c\end{array}$

Subscripts:

opt

counter utilized in impedance design cost function $(1 \leq i \leq N)$

optimum value

pred

design value

\section{Introduction}

As modern turbofan engines have incorporated increased bypass ratios and advanced fan designs, the broadband component of fan noise has grown in relevance. Therefore, while the attenuation of fan tones remains a major factor in engine nacelle acoustic liner design, the ability to simultaneously reduce broadband fan noise levels has become more attractive. With these observations in mind, a process for the design and evaluation of novel broadband acoustic liner concepts with limited fan source information was established in a previous study 1 Specifically, a statistical fan source model was used within an acoustic duct propagation code to predict optimum impedance spectra for the Source Diagnostic Test (SDT) fan (see Figure1a). Acoustic liner modeling tools were then used to identify geometric liner parameters (within manufacturing constraints) necessary to produce impedance spectra that most closely matched the predicted optimum values. Initially, constant-depth, double-degree of freedom (DDOF) designs were explored. In pursuit of enhanced broadband performance, variable-depth, multi-degree of freedom (MDOF) designs were also considered. The impedance spectra for each of these configurations were then used with the propagation code to predict attenuation spectra that could be achieved with each liner. A broadband liner design was produced that was predicted to provide increased attenuation over conventional tonal designs (see for example, Figure $1 \mathrm{~b}$ ) for the full range of frequencies and operating conditions considered. Unfortunately, plans to carry the designs through fabrication, testing, and evaluation did not materialize.

The current work builds upon this previous study by including liner design, testing, and evaluation in the NASA Glenn Advanced Noise Control Fan rig. Additionally, increased computational efficiency and an expanded candidate liner design space were incorporated into the liner design process. The remainder of the paper details these improvements, as well as the resultant broadband liner design and evaluation, and is organized as follows. The selected test configuration and operating conditions of interest are first presented in Section $\Pi$ This is followed by a discussion of the computational approach and implementation in Section [III. The liner design process is then described in Section IV and evaluation of performance in Section $\mathrm{V}$. Finally, concluding remarks regarding some of the more significant results and further areas of interest are presented in Section VI

\section{Test Configuration}

The test bed selected for this study was the Advanced Noise Control Fan (ANCF), a 4-foot diameter low speed fan used for validation of noise reduction concepts. The ANCF, shown in Figure 2a, operates inside an enclosed facility designed such that the ANCF is in an anechoic environment. The nominal operating condition of the ANCF is $1800 \mathrm{RPM}$ (tip speed $375 \mathrm{ft} / \mathrm{sec}$ ) providing an inlet duct Mach number of $\sim 0.15$ and a fundamental blade passing frequency (BPF) of $\sim 500 \mathrm{~Hz}$. The ANCF is comprised of a series of 11 and 12 inch long cylindrical spools that are axially interchangeable, enabling rapid testing of a variety of configurations. As an alternative to the fan source, the Configurable Fan Artificial Noise System (CFANS) may be utilized to generate and control circumferential modal source content. The system consists of rings of 16 circumferentially distributed sets of electromagnetic drivers flushmounted on the inner wall at four axial locations. The ANCF also allows for the insertion of a rotating microphone rake to measure resultant modal content..$^{2]}$ Therefore, for selected test configurations, in-duct rotating rake measurements and farfield acoustics measurements may be obtained. 
Further discussion of the ANCF and full test program may be found in a companion paper ${ }^{[3}$ However, this work focuses on the liner design and evaluation process for an additional configuration used for initial testing. In addition to the standard horizontal configuration, testing was conducted with the ANCF hardware in a vertical orientation to provide a clean, annular duct for experimentation. In this case, the spool pieces were stacked up in a vertical orientation on the floor as shown in Figure $2 \mathrm{~b}$. This removed the support hardware of the standard arrangement, providing the basis for a constant area annular duct. Two such configurations were achieved through the use of two cylindrical centerbodies with constant diameters of $24 \mathrm{in}$. $(60.96 \mathrm{~cm})$ and $36 \mathrm{in}$. $(91.44 \mathrm{~m})$. These correspond to equivalent annular duct hub-to-tip ratios, $\tau$, of 0.5 and 0.75 , respectively. The entire stack rested on the floor, and approximately $6 \mathrm{in}$. of foam material was placed in the bottom of the stack to minimize reflections from the floor. For this noflow configuration, in-duct rotating rake measurements were acquired at the entrance and exit of the lined section to determine measured acoustic liner transmission loss. The CFANS was used to generate individual circumferential modes of order, $m$, ranging from 0 to 6 over a frequency range of 500 to $1500 \mathrm{~Hz}$. In addition, a source field was generated that consisted of all possible modes with equal amplitude and random phases. These phases were set in a deterministic manner (i.e., the same set of random phases was used for each test). In contrast to the specification of individual circumferential modes, this latter source more closely approximates the fully randomized source used in the liner design process (see Section IIII). Therefore, measured transmission loss values for the randomized phase cases are used in the comparisons presented in Section $\mathrm{V}$.

\section{Optimum Impedance Prediction}

The broadband liner design process begins with a series of propagation predictions (at selected flow conditions and frequencies) over a specified impedance design space. Based on a given cost function, these calculations are used to obtain a predicted optimum impedance spectra over the full range of flow conditions of interest. In this work, the CDUCT-LaRC (CDL) duct propagation and radiation code was used to obtain predicted attenuation values. This code calculates the propagation of a given acoustic source ahead of the fan face or aft of the exhaust guide vanes in the inlet or exhaust ducts, respectively. The three-dimensional duct may be acoustically lined (possibly circumferentially and radially segmented) in specified areas and incorporate struts/bifurcations. All of the modules that currently make up the CDL framework have been discussed previously ${ }^{4}$ However, this discussion focuses on the propagation module, as it is most pertinent to this investigation.

The duct propagation module is based on the CDUCT code developed by Dougherty ${ }^{5 / 6}$ and extended by Lan.$^{7 /}$ This code utilizes a parabolic approximation to the convected Helmholtz equation and offers a computationally efficient model that accounts for the complexities of fully three-dimensional nacelle configurations. The CDL code has been extended ${ }^{\sqrt{4}}$ to support multi-block propagation calculations. The grid connectivity is determined and data is transferred from upstream to downstream blocks without user intervention. The output of the propagation module includes the acoustic potential or pressure within the duct, which may be utilized by the radiation module for acoustic radiation calculations.

While CDL can accept arbitrary source specification (i.e., it is not a modal code), it is convenient to specify the acoustic source distribution in terms of duct modes. For situations in which the source pressure is available, this greatly simplifies the conversion to the required acoustic potential. However, when source information is not available, an assumption on the source description must be made. This is generally the case and the approach taken here follows that described by Zlavog and Eversman ${ }^{8 \mid 9}$ in a series of statistical studies into the effects of randomized modal source power and/or phase on attenuation in lined ducts. In this study, the source modal powers (and hence, amplitudes) and modal phases are allowed to vary randomly and independently. Thus, with equal probability of occurrence, the amplitude and phase for each cut-on source mode may take any value in the intervals from 0 to 1 and 0 to $2 \pi$, respectively. This uncertainty in source specification will undoubtedly result in uncertainty in the predicted sound levels. However, as discussed by Zlavog and Eversman, ${ }^{89}$ for the case of all propagating circumferential and radial acoustic modes with random modal power and phase, transmitted power appears to be normally distributed. In their statistical studies, this also produced statistical distributions with the least standard deviation. Therefore, as discussed in a previous CDL study, ${ }^{10}$ Student's t-distribution was used to statistically analyze the predicted attenuation results. For each configuration, 11 simulations were performed to produce a sample population from which mean, $\mu$, and standard deviation, $\sigma$, values can be calculated.

To maintain connectivity with the previous liner optimization study, ${ }^{1}$ in-duct attenuation was chosen as the cost function for the impedance optimization. Thus, the optimum impedance spectrum was predicted using the propagation module of the CDL code with the aforementioned source model. Previously, the maximum in-duct attenuation was obtained by plotting predicted mean attenuation over a specified range of normalized resistance range $0<\theta \leq 5$ and 
normalized reactance range of $-5 \leq \chi \leq 5$. Preliminary estimates were obtained using a coarse grid over the full impedance domain. A fine grid over a sub-domain encompassing the preliminary value was then used to obtain a refined optimum impedance prediction. It is evident that this approach involves a large number of propagation calculations. To alleviate this issue, the current study coupled the CDL calculations with optimization routines. This was accomplished by using the Python programming language (http://www.python.org) to "wrapper" the CDL calculations. The use of the Python scripts allow access to the full set of tools available in the optimization package of the Scipy $\frac{111}{1}$ library. Thus, predicted optimum impedance spectra (over a larger design space encompassing $0<\theta \leq 10$ and $-10 \leq \chi \leq 10$ ) were obtained with a large reduction in computational effort.

Optimum impedance predictions were obtained for frequencies ranging from $500 \mathrm{~Hz}$ to $1500 \mathrm{~Hz}$ in $100 \mathrm{~Hz}$ increments with zero mean flow. Initial calculations focused on the $\tau=0.5$ vertical configuration and showed that only modest attenuation could be achieved at the given hub-to-tip ratio. As shown by Kraf ${ }^{12}$ and Rice $\frac{\sqrt{13}}{\sqrt{12}}$ this is to be expected due to the ratio of liner axial length to duct height $(L / H)$ and frequencies of interest. While this provided data for further validation of the broadband liner design process, configurations allowing for larger liner benefits were desired. Therefore, based on further predictions and the versatility of the vertical test set-up, the $\tau=0.75$ case was included in testing. This provided additional validation data, as well as a configuration for which larger liner attenuation could be achieved. Ultimately, the predicted optimum impedance for the $\tau=0.5$ case was selected as the design target. This was mainly due to the fact that the constant area section of the standard ANCF configuration has a 24 inch centerbody. Also, the difference between the predicted optimum impedance spectra between the two cases was minimal.

\section{Liner Modeling and Fabrication}

As indicated above, the CDL propagation code was used to determine target optimum impedance values within the duct. The efficiency of this process allows for parallel-element, variable-impedance, broadband liner concepts 14 at various technology readiness levels (TRL) to be considered for implementation. Prediction tools based on the combination of a transmission line calculation $\frac{15}{15}$ and the Two-Parameter Impedance Prediction Mode 16 are used to model the resultant liner impedance spectra. To achieve broadband performance, the liner consisted of a multi-layer configuration incorporating septa (or "mesh-caps") embedded into a honeycomb core.14 This concept allows the acoustic liner to be customized such that the surface impedance of each individual cell is independently controlled. This is achieved by the combination of parameters used to set the impedance in each cell. The various cells are then arranged into a grid pattern of different mesh-cap depths or resistances within the acoustic panel to achieve a desired distributed impedance.

Clearly, manufacturing constraints (e.g., liner geometric parameters such as porosity and core depth) are key ingredients in this modeling phase, and must be taken into account to design acoustic liners that can be realistically achieved. In this study, the honeycomb cells were restricted to contain one mesh-cap. The mesh-cap depth, as well as its DC flow resistance, were allowed to vary from cell to cell. Representative liner configurations resulting from mesh-cap insertion are shown in Figure 3 to illustrate the two-layer setup and geometrical parameters. Finally, based on currently available manufacturing techniques, the grid pattern for the MDOF liner was limited to a configuration of four cells as shown in Figure 4

As described in Section III the optimization process using CDL in-duct propagation results provides predicted optimum impedance values, $\zeta_{o p t, i}$, at a specific flight condition and frequency. The liner modeling tools are then used to obtain design impedance values, $\zeta_{\text {pred }, i}$, that minimize the function

$$
F=\left\{\sum_{i=1}^{N} W_{i}\left(\zeta_{o p t, i}-\zeta_{p r e d, i}\right)\left(\zeta_{o p t, i}-\zeta_{p r e d, i}\right)^{*}\right\}^{0.5} .
$$

Here, the values $W_{i}$ are weighting values that may be used to assign increased importance to a selected number of frequencies and/or flow conditions and $N$ is the total number of frequency/flow condition combinations included. Note that with $W_{i}=1$ for all $N$, equation 1 reduces to the $L_{2}$ norm of the difference between the predicted optimum and the design impedance values and was the approach used in this study.

Based on the above cost function, a constant-depth, double-degree of freedom (DDOF) and a variable-depth, multi-degree of freedom (MDOF) liner were designed and manufactured. Due to the relatively low target optimum resistance values, a 20 MKS Rayls wiremesh facesheet was selected for both liners. This minimized the impact of the facesheet on the overall resistance and allowed for greater flexibility in tuning the resistance of individual cells via the embedded mesh-caps. The liner core is comprised of a 0.375 -inch $(0.953 \mathrm{~cm})$ diameter hexagonal-shaped honeycomb 
core with an overall depth of 2.0 inches $(5.08 \mathrm{~cm})$. Mesh-cap materials ranging in DC flow resistance values from 600 to 1200 MKS Rayls were considered. Additionally, the distance between the mesh-cap and liner backplate, $h_{1}$, was allowed to vary (see Figure 3). In the design process, this value ranged from 0.20 in $(0.508 \mathrm{~cm})$ to 1.80 in $(4.57 \mathrm{~cm})$ at 0.20 in $(9.508 \mathrm{~cm})$ increments. An optimization process was used to determine the best combination of geometrical parameters to achieve the desired impedance values. For the DDOF liner, the design was constrained to a single constant mesh cap resistance and insertion depth for all cells. Conversely, for the MDOF liner, a four-cell combination of mesh cap resistance and insertion depth (see Figure 4) was utilized.

The final core designs for both liners were fabricated at Hexcel's Research and Technology facility in Casa Grande, AZ. These were incorporated into an existing spool piece having a 48 inch inner diameter, an effective axial length of 16 inches, and approximately 4 inch depth. A photograph of the empty spool piece is shown in Figure 5a. The liner assembly (see Figure 6) was built up starting from the outer radius and working inward. In order to provide a compression mechanism for the entire assembly, two layers of carpet padding were first laid in the spool. This accounted for geometric imperfections in the spool wall and allowed for a tight fit. A hard rubber sheet was then inserted to provide a seal at the base of the honeycomb core and prevent acoustic leakage between adjacent cells. One of the two liner cores was then placed on top of this rubber sheet, followed by the wire mesh facesheet (DC flow resistance of 20 MKS Rayls). Finally, a coarse screen covering was fastened to the facing of the inner ledge of the spool frame to compress the entire lay-up securely and provide a flush flow surface in the duct. A photograph of an installed liner assembly is shown in Figure $5 \mathrm{~b}$.

The final designs for both liners attempted to account for this assembly process. For example, the predicted compliance of the rubber backing was taken into account, rather than assuming a rigid backplate. However, consideration of the coarse screen was more problematic. At the time of design, the resistance of the coarse screen was assumed to be negligible. However, after core fabrication, it was estimated that, based on approximate open area, the screen would increase resistance by $0.5 \rho c$. The predicted impedances for both liners were therefore increased by this amount and carried through pre-test predictions, the results of which are shown in Figures $7 \mathrm{a}$ and $7 \mathrm{~b}$. It can be seen that the character of the target impedance (relatively low resistance at lower frequencies and decrease in reactance with increasing frequency) make it difficult to match with a physically-realizable liner. Taking the adjustment for the coarse screen into account, the design resistance values are well above the optimum value at the lower frequencies. Additionally, the $-\cot (k d)$ behavior evident in the design reactance spectra is counter to the optimum reactance trend. The predicted mean attenuation associated with the optimum, DDOF, and MDOF impedance spectra are shown in Figures $8 \mathrm{a}$ and $8 \mathrm{~b}$. respectively. Due to the statistical nature of the source, error bars representing the $95 \%$ confidence intervals for the mean attenuation are also included in these figures. The issues with low achievable attenuation discussed previously are visible in Figure $8 \mathrm{a}$, although the upper bounds of the $95 \%$ confidence intervals indicate the possibility of attenuation values close to $6 \mathrm{~dB}$ at some frequencies. The difficulty in matching the optimum impedance at the low frequencies is also apparent, as the predicted attenuation values for the DDOF and MDOF liners are well below those predicted for the optimum. The increased attenuation resulting from the larger centerbody (and hence $L / H$ ) are evident in Figure $8 \mathrm{~b}$. This also represents a case in which the possible advantage of the MDOF approach may not be realized. In this situation, the ability to achieve a small increase in attenuation at higher frequencies with an associated larger decrease in attenuation at the lower frequencies is probably not cost effective. However, this illustrates the value of the design process in concurrently evaluating various liner designs (i.e., DDOF, MDOF, etc.) and their relative costs/benefits. It should also be noted that a main goal of validating the efficacy of the design process through design, fabrication, and evaluation remains valid via comparison of the predicted performance with measured results.

\section{Liner Performance Evaluation}

In order to evaluate the liner performance and provide further confidence in the liner design process, comparisons between predicted and measured transmission loss are performed. The measured values are obtained from the aforementioned rotating rake data which, based on previous experience, are assumed to have a measurement error of $+/-1$ $\mathrm{dB} \cdot \sqrt[3]{3}$ The predicted values are those presented in Section IV with error bars representing the $95 \%$ confidence intervals for the mean attenuation. The DDOF liner was tested only in the $\tau=0.5$ configuration, for which comparison of measured and predicted attenuations are presented in Figure 9a. The MDOF liner was tested in both configurations $(\tau=0.5,0.75)$ and corresponding comparisons are provided in Figure $9 \mathrm{~b}$

The prediction of lower achievable attenuations for the $\tau=0.5$ case is confirmed in the measured data. However, the predictions under-predict the measured values at several frequencies. A similar result is seen in the $\tau=0.75$ configuration, for which the MDOF liner predictions are below the measured values, but capture the measurement trend. It is interesting to note that the location of maximum attenuation observed in both the predictions and measurements 
corresponds closely with the frequency $(\sim 1100 \mathrm{~Hz})$ at which the design and optimum reactance values are most closely matched (see Figure $7 \mathrm{~b}$ ).

The general observations drawn from these comparisons agree with those from previous studies (see, for example, $\frac{17}{17}$, in which the CDL code provided conservative attenuation predictions that correctly captured measured trends. However, for the $\tau=0.75$ case in particular, the magnitude of the under-predictions in the current study appears to be larger than in previous applications. Upon further investigation, a number of possible sources for the discrepancy were identified, including:

- Centerbody compliance: Each centerbody was constructed with thick cardboard (nominally 0.25 in thick). Testing of this material indicated that it was quite rigid, suggesting that this effect is minimal. Nevertheless, due to the low attenuation values for the $\tau=0.5$ case, assigning a small compliance to the centerbody may lead to a slight improvement in attenuation comparisons.

- Reflections: The efficiency gained by the parabolic approximation in the CDL code comes at the expense of reduced accuracy when reflection of acoustic waves in the axial direction becomes important, as these effects are not captured in the formulation. Reflections are possible in the test set-up due to impedance discontinuities at the leading and trailing edges of the liner, flanges between adjoining spool pieces, as well as the duct terminations and may lead to some discrepancy.

- Source description: The predictions incorporate a fully randomized modal source model, whereas a single, phase-randomized, but fixed, distribution of modes was used in the experimental set-up. As the attenuation is dependent on source content, this difference in source content will affect the quality of the comparison.

- Coarse screen resistance: As mentioned previously, the predicted resistances for both the DDOF and MDOF liners were increased by $0.5 \rho c$ to account for the presence of the coarse screen. This value was based on testing of a similar sample comprised of a coarse screen bonded to a wiremesh facesheet. Additional tests were conducted after completion of the ANCF test and indicate this increase should be much closer to zero, although work to more precisely determine the value is ongoing.

As an updated value for the coarse screen resistance was not available at the time of this study, additional CDL predictions were performed in which the presence of the coarse screen was assumed to be negligible (i.e., the predicted resistance values for both liners were reduced by $0.5 \rho c$ ) to investigate this effect further. This essentially bracketed the possible coarse screen resistance value and provided an lower/upper bound on the predicted attenuation. Comparisons of the complete set of predictions with the measured attenuation values for the DDOF and MDOF liners in the $\tau=0.5$ configuration are shown in Figures $10 \mathrm{a}$ and $10 \mathrm{~b}$, respectively. The updated results $(\theta-0.5)$ show a slight increase in the predicted attenuation, as well as the $95 \%$ confidence intervals. The larger confidence intervals may indicate a need for further investigation of the difference in source specification between the predictions and measurements. However, neglecting the acoustic effects of the coarse screen appears to provide a general improvement in the comparison between predicted and measured values. More convincing evidence is found in the comparisons for the $\tau=0.75$ configuration presented in Figure 11. In this case, neglecting the acoustic effects of the coarse screen provides a larger increase in the predicted peak attenuation and clearly improves the comparison between predicted and measured values. Note also that the predicted frequency of peak attenuation is unchanged, which is to be expected as the acoustic effects of the coarse screen are manifested through changes in the resistance. Therefore, the updated results point to a need to more accurately quantify the effects of the coarse screen on design impedance. Perhaps more importantly, the findings also provide further evidence to support the expectation that the CDL code can be used to investigate various liner configurations and provide conservative attenuation predictions that correctly capture measured trends.

\section{Concluding Remarks}

This paper describes improvements to a previously established process for the design of novel broadband acoustic liner concepts (e.g., DDOF, MDOF, etc.) with limited fan source information. The selection of the ANCF rig afforded the opportunity to include liner fabrication and testing to further validate the broadband liner design process. Predictions on the initial test configuration indicated an optimum impedance spectrum that was difficult to achieve, accompanied by relatively low achievable attenuation values. However, the versatility of the vertical test set-up allowed for an additional configuration, for which larger attenuation values could be achieved, to be included in the test matrix. Early stages of the design process also showed the ANCF to represent a case in which the possible advantage of the MDOF approach may not be realized. In comparison with the DDOF design, the MDOF design showed a small 
increase in attenuation at higher frequencies with an associated larger decrease in attenuation at the lower frequencies that may not be cost effective. This is, of course, configuration dependent and illustrated the value of the design process in concurrently evaluating various liner designs (i.e., DDOF, MDOF, etc.) and their relative costs/benefits. In addition, a main goal of validating the efficacy of the design process through design, fabrication, and evaluation of various liner designs was met.

Comparisons of predicted and measured attenuation values were ultimately seen to follow similar trends to those identified in previous studies, providing further confidence in the conclusions drawn. However, through the course of this study, a number of opportunities for further research were also identified. First, the difference in source specification between predictions and measurement was identified as a possible source of discrepancy in the associated attenuation values. Information on the actual source content in the measured data is available through rotating rake data. Therefore, further predictions will be performed using this source information to quantify the effect of source specification on predicted attenuation. Additionally, the likelihood of a non-rigid centerbody affecting the predicted attenuation may also be considered through these calculations. Finally, further investigation of the predicted contribution of the coarse screen to the liner assembly impedance is of interest. Comparison of predicted and measured attenuation values clearly showed it to have an effect. Therefore, testing of low-resistance wiremesh facesheets bonded to representative coarse screens will be conducted to better quantify the interaction. This will be critical to properly utilizing the full set of test data for continued validation of the design tools.

In addition to bringing these areas of interest to light, this study provided further evidence to support the expectation that the CDL code can be used to investigate various liner configurations and provide conservative attenuation predictions that correctly capture measured trends. It has also provided an excellent opportunity to improve the integrated use of duct acoustic propagation/radiation and liner modeling tools for broadband liner design.

\section{Acknowledgments}

This research was funded by the Fixed Wing Project of NASA's Fundamental Aeronautics Program.

\section{References}

\footnotetext{
${ }^{1}$ Nark, D. M. and Jones, M. G., "Broadband Liner Optimization for the Source Diagnostic Test Fan,” AIAA Paper 2012-2195, 2012.

${ }^{2}$ Sutliff, D. L., "Turbofan Duct Mode Measurements Using a Continuously Rotating Microphone Rake," International Journal of Aeroacoustics, Vol. 6, No. 2, 2007, pp. 147-170.

${ }^{3}$ Sutliff, D. L., Jones, M. G., and Nark, D. M., "In-Duct and Far-field Experimental Measurements from the ANCF for the Purpose of Improved Broadband Liner Optimization," 20th AIAA/CEAS Aeroacoustics Conference, American Institute of Aeronautics and Astronautics, Reston, VA (submitted for publication), 2014.

${ }^{4}$ Nark, D. M., Farassat, F., Pope, D. S., and Vatsa, V., "The Development of the Ducted Fan Noise Propagation and Radiation Code CDUCTLaRC," AIAA Paper 2003-3242, 2003.

${ }^{5}$ Dougherty, R. P., “A Wave-Splitting Technique for Nacelle Acoustic Propagation,” AIAA Paper 97-1652, 1997.

${ }^{6}$ Dougherty, R. P., “A Parabolic Approximation for Flow Effects on Sound Propagation in Nonuniform, Softwall, Ducts," AIAA Paper 99$1822,1999$.

${ }^{7}$ Lan, J. H., “Turbofan Duct Propagation Model,” NASA CR 2001-211245, 2001.

${ }^{8}$ Zlavog, G. and Eversman, W., "Source effects on attenuation in lined ducts. Part I: A statistically based computational approach," Journal of Sound and Vibration, Vol. 307, No. 1-2, 2007, pp. 113-138.

${ }^{9}$ Zlavog, G. and Eversman, W., "Source effects on attenuation in lined ducts. Part II: Statistical properties," Journal of Sound and Vibration, Vol. 307, No. 1-2, 2007, pp. 139-151.

${ }^{10}$ Nark, D. M., “Assessment of Radiated Fan Noise Prediction Capabilities using Static Engine Test Data," AIAA Paper 2011-2807, 2011.

${ }^{11}$ Jones, E., Oliphant, T., Peterson, P., et al., "SciPy: Open Source Scientific Tools for Python," 2001 -.

${ }^{12}$ Kraft, R. E., Theory and Measurement of Acoustic Wave Propagation in Multi-Segmented Rectangular Flow Ducts, Ph.D. thesis, University of Cincinnati, 1976.

${ }^{13}$ Rice, E. J., "Modal Propagation Angles in Ducts with Soft Wall and Their Connection with Suppressor Performance," AIAA Paper 79-0624, 1979.

${ }^{14}$ Jones, M. G., Howerton, B. M., and Ayle, E., "Evaluation of Parallel-Element, Variable-Impedance, Broadband Acoustic Liner Concepts," 18th AIAA/CEAS Aeroacoustics Conference, American Institute of Aeronautics and Astronautics, Reston, VA (submitted for publication).

${ }^{15}$ Parrott, T. L. and Jones, M. G., "Parallel-Element Liner Impedances for Improved Absorption of Broadband Sound in Ducts," Noise Control Engineering Journal, Vol. 43, No. 6, 1995, pp. 183-195.

${ }^{16}$ Jones, M. G., Parrott, T. L., and Watson, W. R., "Uncertainty and Sensitivity Analyses of a Two-Parameter Impedance Prediction Model," AIAA Paper 2008-2928, 2008.

${ }^{17}$ Nark, D. M., Envia, E., and Burley, C. L., “On Acoustic Source Specification for Rotor-Stator Interaction Noise Prediction,” AIAA Paper 2010-3713, 2010.
} 


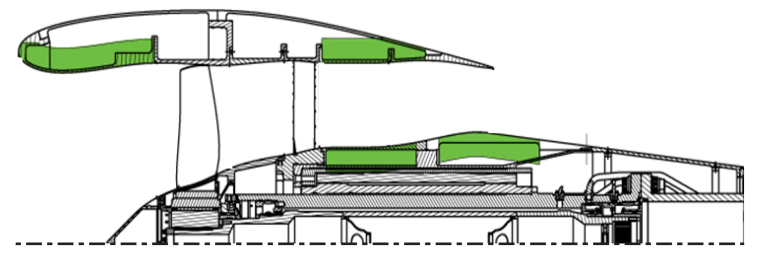

(a) SDT model fan stage in baseline configuration with acoustic liner locations denoted in green.

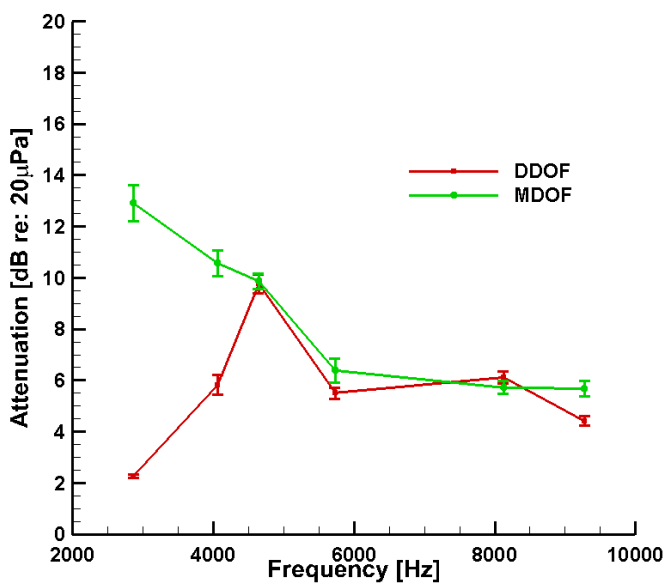

(b) Predicted attenuation at a representative flight condition.

Figure 1: Illustration of predicted MDOF liner benefit over a conventional DDOF design for the SDT Fan. Error bars indicate $95 \%$ confidence intervals.

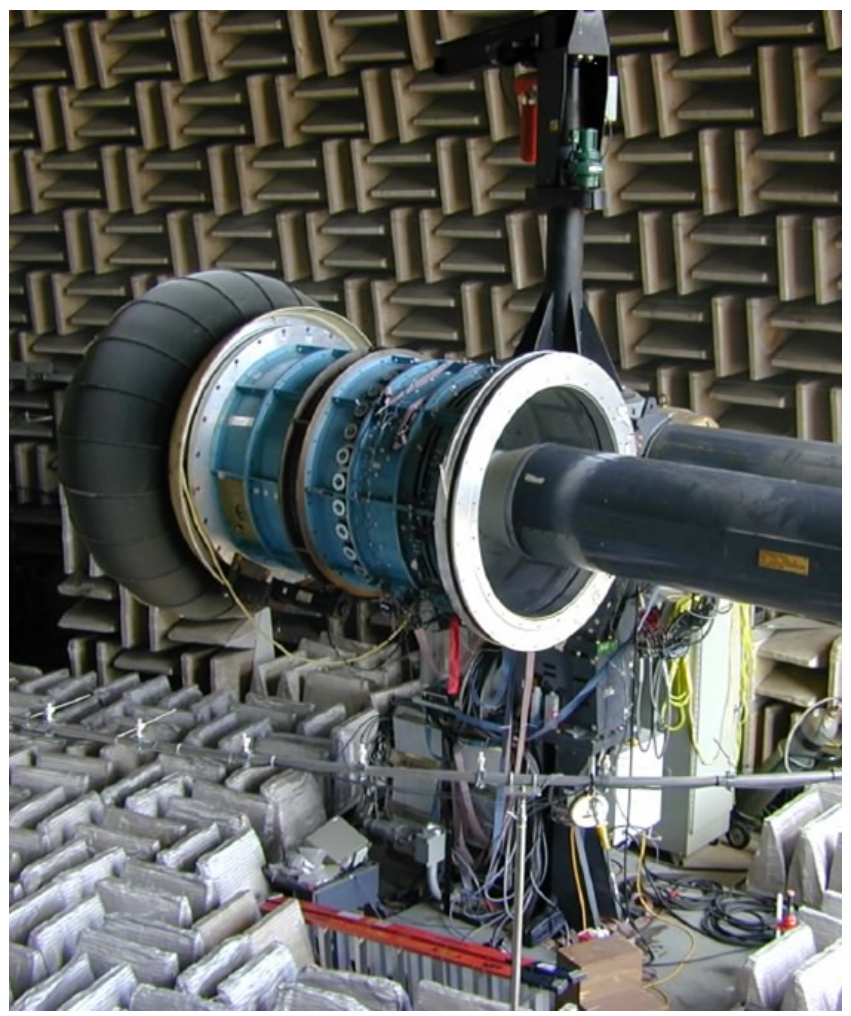

(a) Standard ANCF configuration.

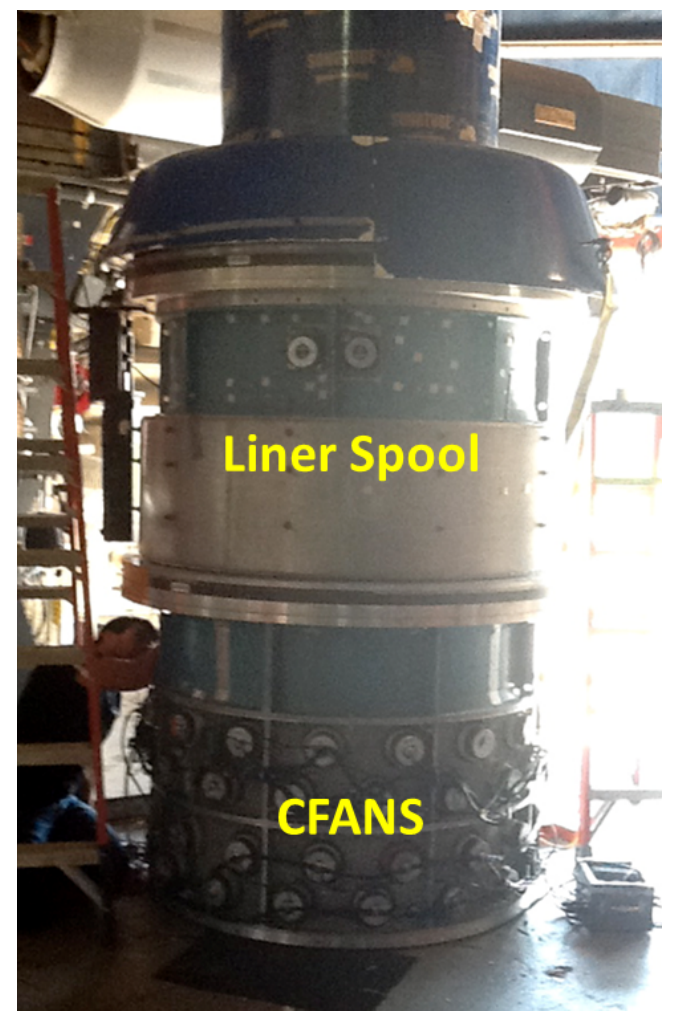

(b) Stack-up in vertical, off-stanchion orientation (No Flow).

Figure 2: Advanced Noise Control Fan (ANCF) rig configurations 


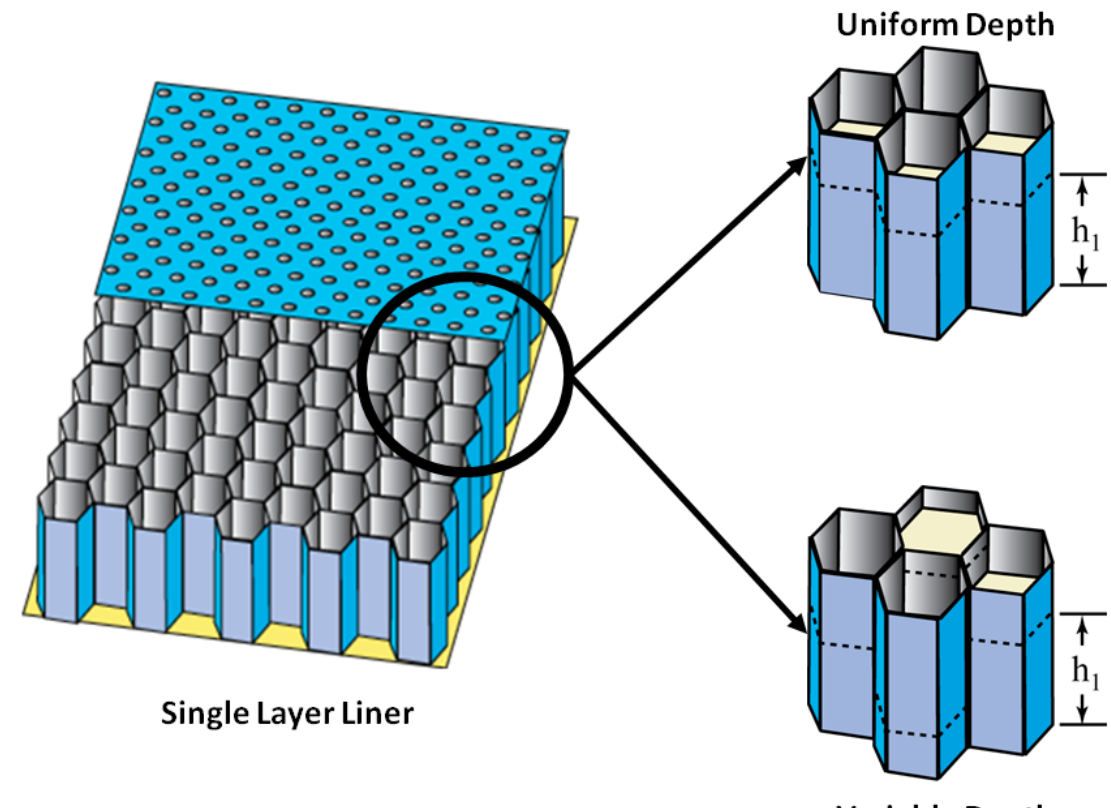

Variable Depth

Figure 3: Parent single layer liner and resultant two-layer liner configurations (uniform and variable depth) via meshcap insertion.

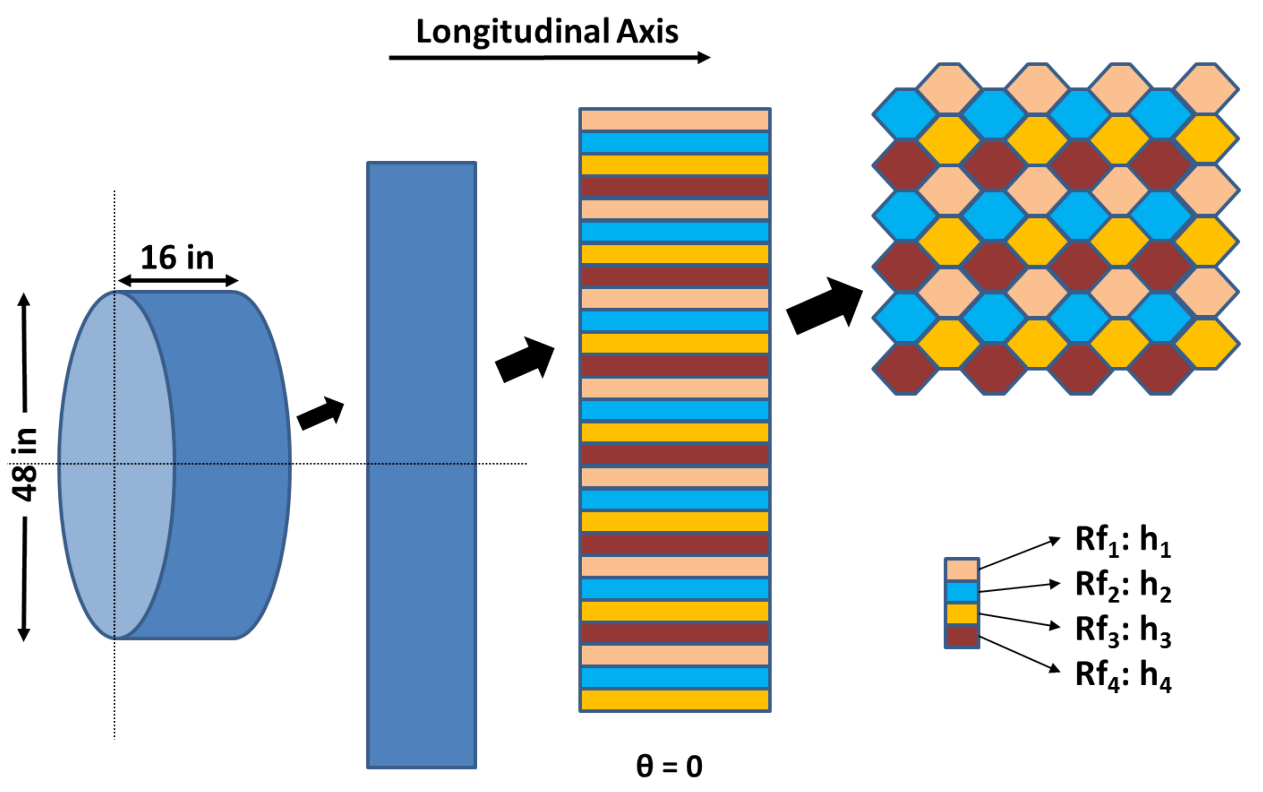

Figure 4: Four-cell mesh-cap insertion pattern for variable depth liner. 


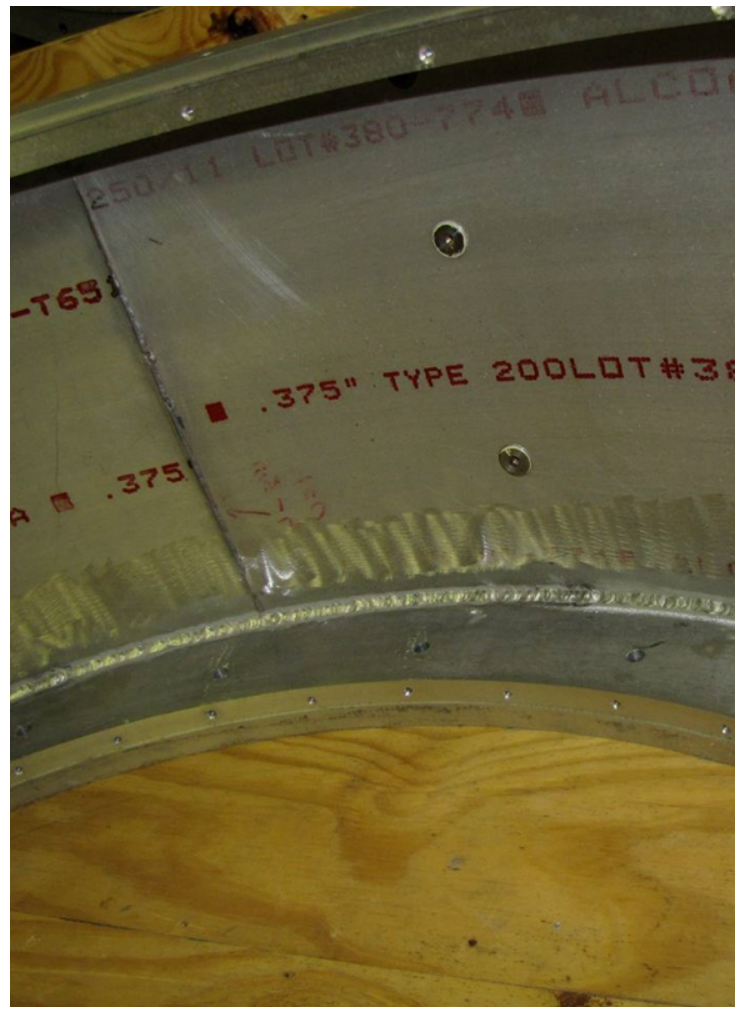

(a) Liner spool piece with empty cavity.

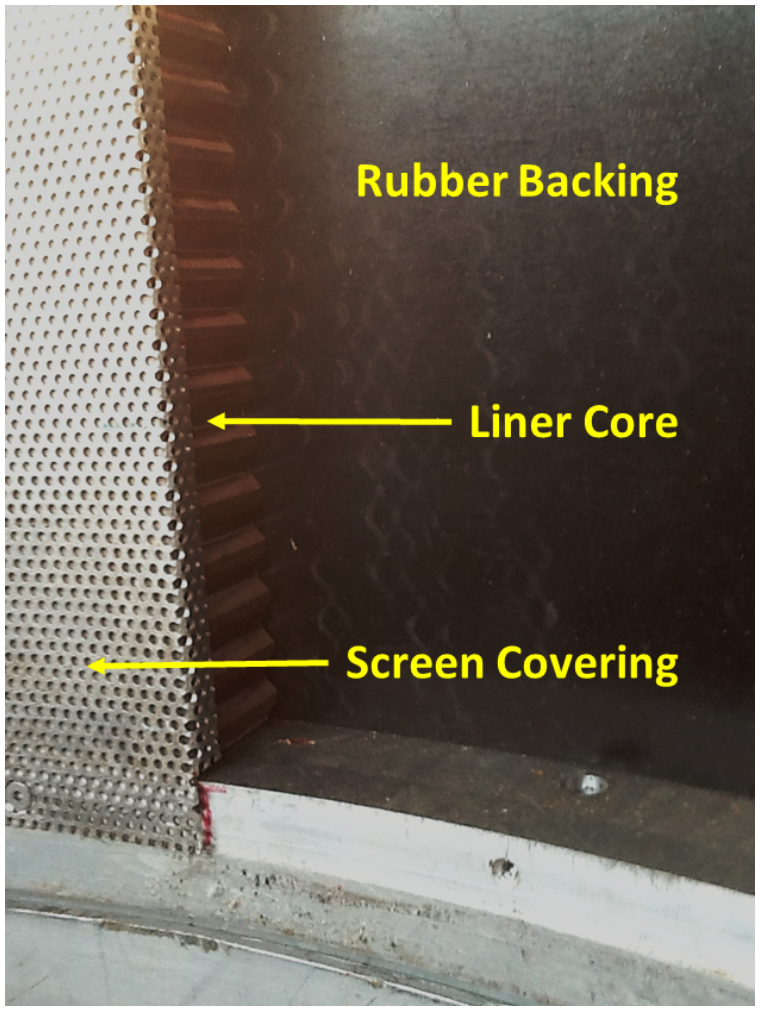

(b) Complete liner spool build-up.

Figure 5: Photographs of the liner assembly process.

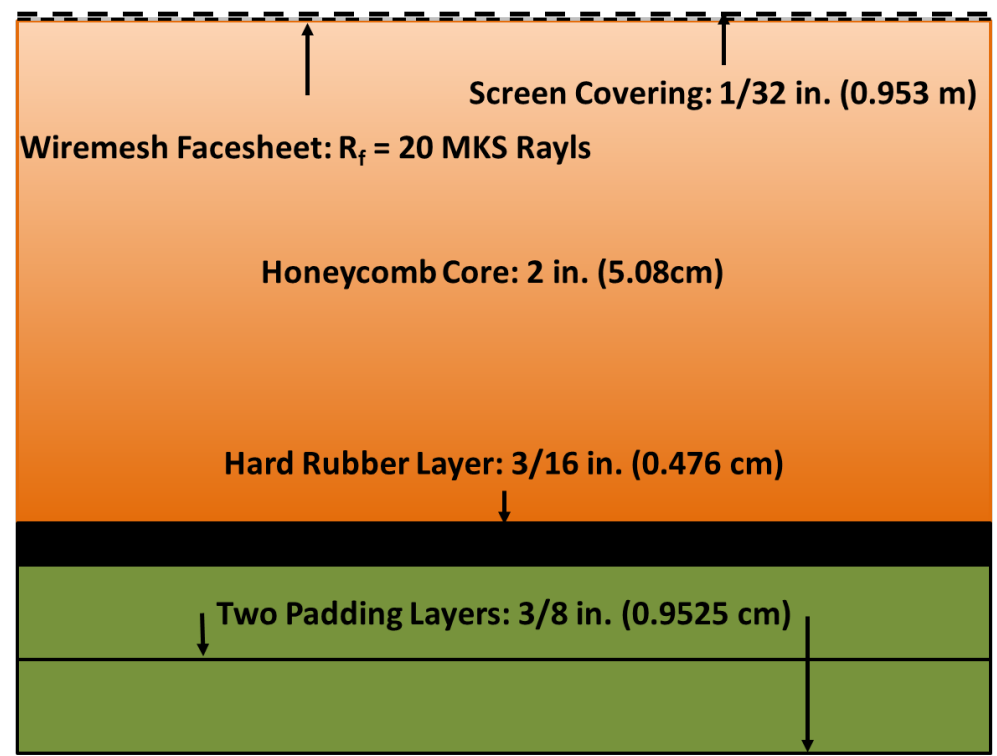

Figure 6: Cross-section of liner assembly. 


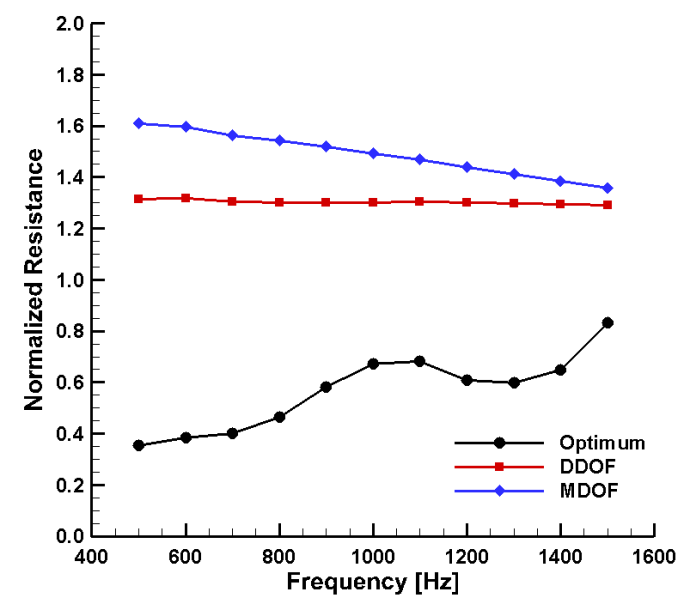

(a) Normalized Resistance.

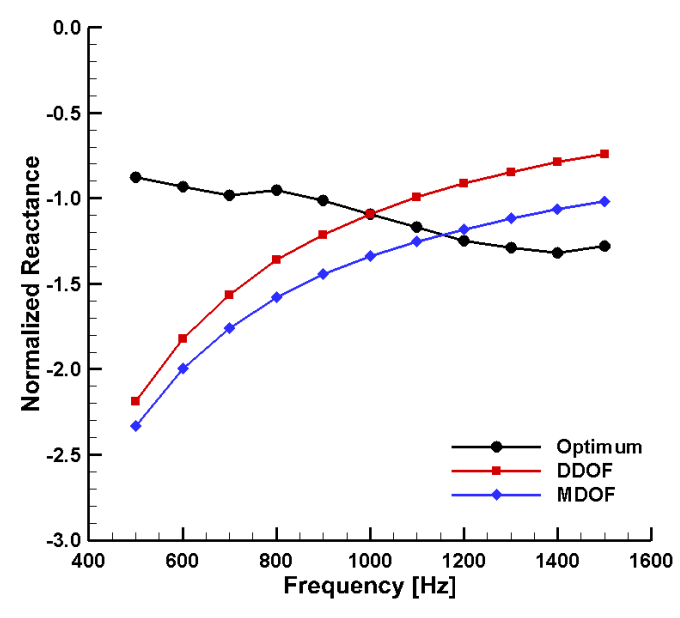

(b) Normalized Reactance.

Figure 7: Comparison of optimum and build impedance spectra for the 24 inch diameter centerbody $(\tau=0.5)$.

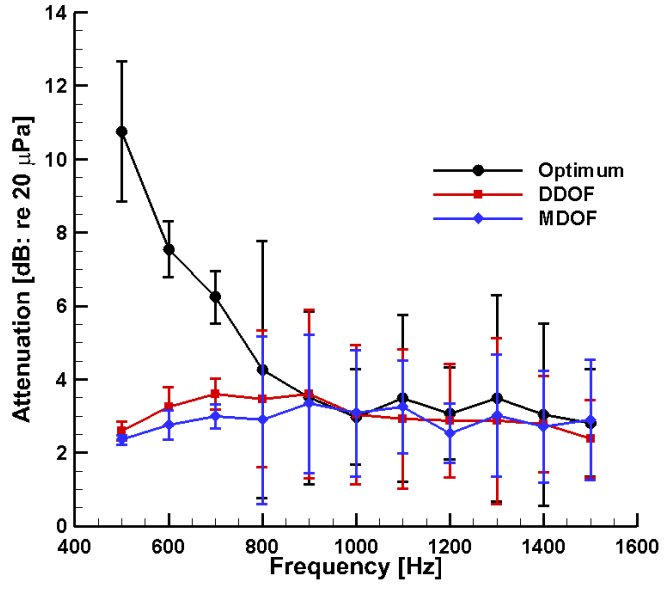

(a) $\tau=0.5(L / H=0.7)$.

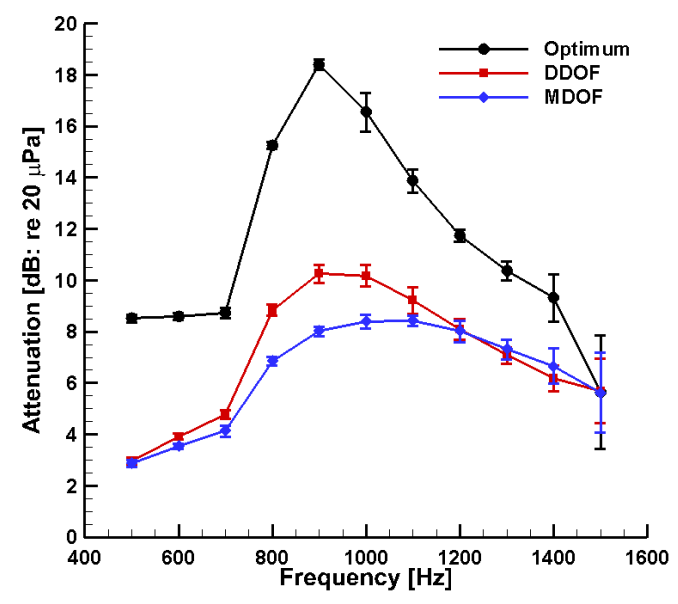

(b) $\tau=0.75(L / H=1.3)$.

Figure 8: Comparison of predicted attenuation values for the two test centerbody configurations. Error bars indicate 95\% confidence intervals on the predicted attenuation values. 


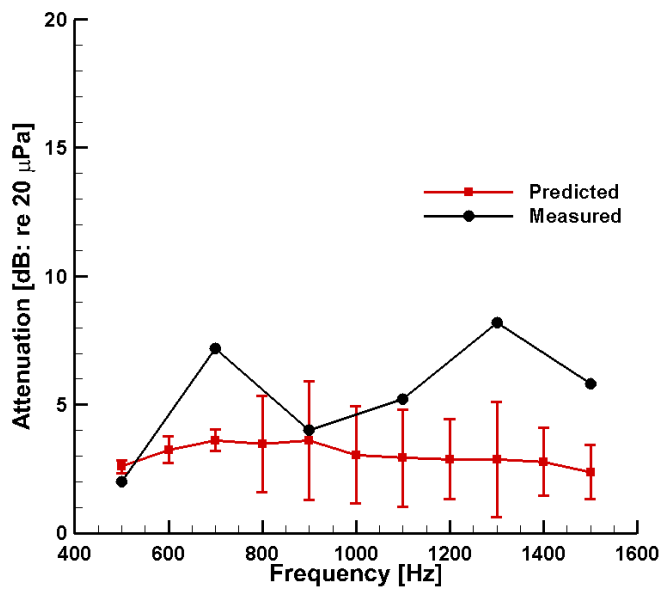

(a) DDOF liner.

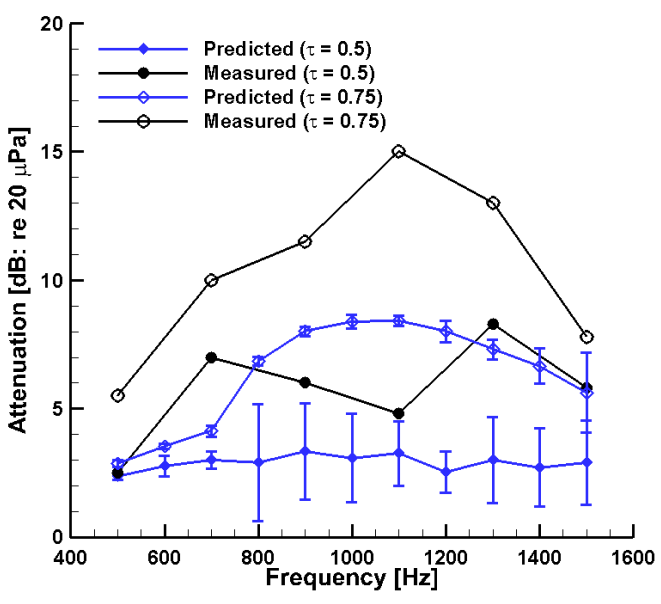

(b) MDOF liner.

Figure 9: Comparison of predicted and measured attenuation values for the DDOF and MDOF liners. Error bars indicate $95 \%$ confidence intervals on the predicted attenuation values.

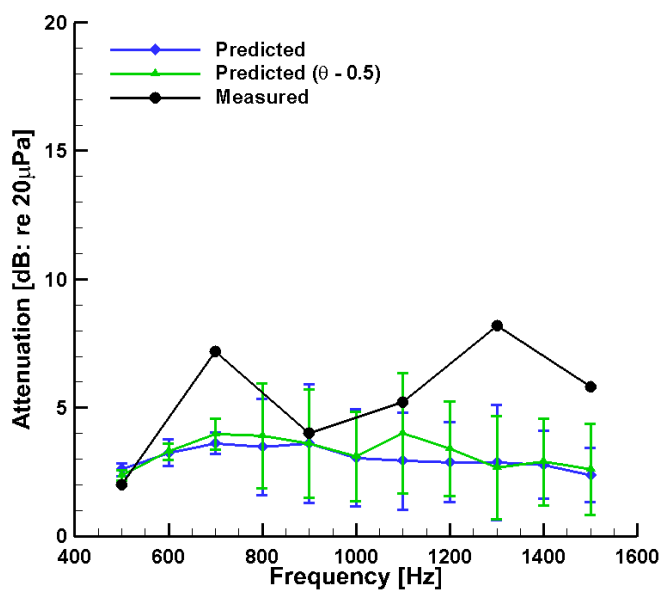

(a) DDOF liner.

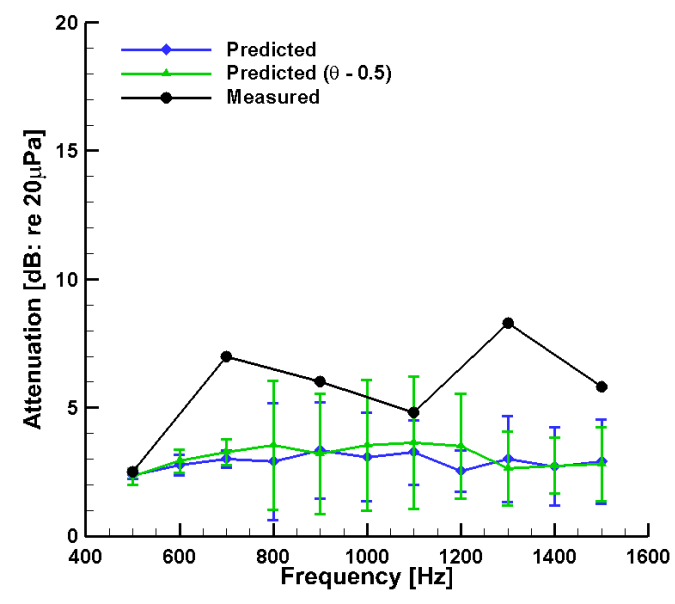

(b) MDOF liner.

Figure 10: Updated comparison of predicted and measured attenuation values for the DDOF and MDOF in the $\tau=0.5$ configuration . Error bars indicate $95 \%$ confidence intervals on the predicted attenuation values. 


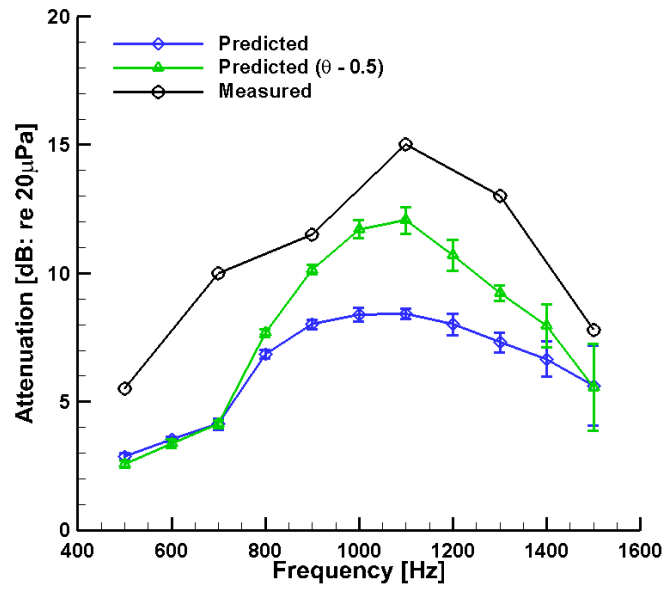

Figure 11: Updated comparison of predicted and measured attenuation values for the MDOF liner in the $\tau=0.75$ configuration . Error bars indicate $95 \%$ confidence intervals on the predicted attenuation values. 